\title{
Buchbesprechungen - Book Reviews - Livres Nouveaux
}

Biological Organisation Cellular and Sub-cellular. Proceeding of a Symposium Organised on Behalf of U.N.E.S.C.O. Edited by C. H. Waddington. Pergamon Press, London 1959. XVIII + 328 p., 70 s.

Ein von C. H. Waddington iiberaus sorgsam und klug vorbereitetes Symposium iiber die zelluliire und subzellulare biologische Organisation, das 1957 in Edinburgh stattgefunden hatte, fand seinen Niederschlag in einem ebenso anregenden wie in-haltsreichen Buch. Es sind in ihm enthalten die kurzen Referate und Mitteilungen iiber die neuesten eigenen Experimente, die gleichsam als Vorspiel zur ebenfalls publizierten ausgiebigen Diskussion iiber die interessantesten Probleme der biolo-gischen Organisation dienen. Die Tatsache, daB die beteiligten Wissenschaftler aus der Schule plaudern, ihre Zweifel, Ansichten und Schwierigkeiten freimiitig zur Diskussion stellen, bereichert das vorliegende Werk, um so mehr, als fiihrende Embryologen, Entwicklungsphysiologen und Zytologen wie Bracket, Callan, Lehmann, Nieuwkoop, Pavan, Pollock, Pontecorvo, Ris, Sjostrand, Toivonen, Waddington (um nur einige Namen zu nennen) an Diskussion und Referat teilnehmen.

Diskutiert werden die Natur der biologischen Organisation, Probleme der Organisation im Bereich des Gens und der Chromosomen, die Wechselwirkung zwischenKern und Zytoplasma (unterstiitzt von elektronen-mikroskopischen Aufnahmen), chemische Aktivitat des Zytoplasmas und die chemische Organisation in der Zelle.Nicht minder interessant sind die Beitrage iiber die embryonale Induktion, dasWerden der Gewebe und der Organe sowie Wachstumsprozesse. Das SchluBkapitelfiihrt in seiner Betrachtung der Mitose wieder zum Verhalten von einzelnen Zellenzuriick. Das Buch wird dem Wunsche seines Herausgebers vollkommen gerecht, namlich die Spannung und die Faszination wiederzugeben, welche das Zusammen-treffen verschiedener Meinungen und experimenteller Gebiete von aktiven For-schern ausstromt. Hedi FritzNiggli, Zurich

Neoplastic Disease at Various Sites. Ed. by D. W. Smithers. Introductory Volume: A clinical prospect of the cancer Problem by D. W. Smithers. E. and S. Livingstone Ltd., Edinburgh and London 1960.

Smithers ist der Herausgeber einer Serie von Monographien iiber Geschwiilsteder verschiedensten Art und Lokalisation. Der 1. Band gibt eine ausgezeichneteUbersicht iiber das Geschwulstproblem iiberhaupt unter weitgehender Beriick-sichtigung der einschlagigen Literatur. Ich kann die Arbeit bestens empfehlen, InVorbereitung sind weitere Bande iiber den Prostatakrebs, iiber Hirngeschwiilste, iiber Larynx- und Pharynxtumoren usw. Fur den Krebsforscher, den Krebs-diagnostiker und den Krebstherapeuten ist der vorliegende 1. Band auBerordentlichwertvoll. Auch die Ausstattung ist vorziiglich. H. R. Schinz, Zurich Medizinische Grundlagenforschung. Band III. Hgb. von F. Bauer et al. G. Thieme, Stuttgart 1960. 754 S., 205 Abb., 47 Tab. DM 178.-.

Jeder Forscher und jeder Arzt sucht vor der gewaltigen Vermehrung des Einzelwissens nach einem guten Uberblick iiber groBe Teilgebiete, um seine Speziali-tat in das Ganze einzugliedern. 
Diesem Zwecke dient auch Band 3 der medizinischen Grundlagenforschung. Es ist klar, daB dabei jene Gebiete vor allem beriicksichtigt

Buchbesprechungen - Book Reviews - Livres Nouveaux 325

werden, die «modern» sind, modern im Sinne einer neuen Methodik oder im Sinne gewonnener neuer Einsichten. Die bearbeiteten Themata beweisen dies. Den Krebs-therapeuten interessiert vor allem das Kapitel iiber die Grundlagen und Ergebnisse der in-vivo-Messung radioaktiver Substanzen im menschlichen Organismus (Mehl) und die Darstelhmg der Strahlenschutzprobleme in arztlicher Sicht (Barth und Frik). Hochmodern ist das Kapitel iiber das EiweiBmolekiil (Turba und Zillig) und die Darstellung des neuesten Standes der Plasmaproteinforschung (Schultze und Heide).

Mein Ziircher Kollege Leuthardt berichtet in Zusammenarbeit mit Stuhlfaut iiberbiochemische, physiologische und klinische Probleme des Fructose-Stoffwechsels.AUfallige Zusammenhange zwischen Fettstoffwechsel und Atherosklerose erortert ineinem englisch geschriebenen Aufsatz Kinsell aus Kalifornien. Die iibrigen Kapitelstammen wieder von deutschen Autoren oder sind wenigstens deutsch verfaBt. DerBotaniker Zimmermann in Tubingen schildert unser heutiges Wissen von der Evolution. Alle Aufsatze sind ausgezeichnet bebildert, und die buchtechnische Aus-stattung ist ganz vorziiglich. Wir empfehlen den Band sehr zur Anschaffung durchBiologen, Arzte und Bibliotheken. $\quad$ H. R. Schinz, Zurich

Raven, Ronald W'.: Cancer. Progress Volume 1960. Butterworths, London 1960. XVI+ 258 p., ill. 60s.

Raven ist weitherum bekannt als Herausgeber eines 6bandigen Werkes iiber den Krebs, das die neuesten Ergebnisse zusammenfaBt. Der vorliegende Band iiber die Fortschritte auf diesem Gebiete im Jahre 1960 fasse ich als Erganzung zu diesem Werke auf. Es enthalt 32 Beitrage, darunter 3 aus dem experimentellen Gebiete, z. B. eine Darstellung der neuesten Erkenntnisse auf dem Gebiet der Tumorvirologie von Harris, 7 Aufsatze sind pathologisch-anatomischer Natur. Stewart berichtet iiber Carcinomzellen im stromenden Blut, Dawson iiber das Brustcarcinom, Spencer iiber Carcinoma der Nebenschilddriise. Unter den statistischen Arbeiten erwahne ich eine kurze Darstellung iiber Rauchen und Krebs von Wynder und Hoffmann. Uber Berufskrebse referiert Bidsdrup, Strickland schildert die Ergebnisse der Arteriographie bei Tumoren der Knochen und der Weichteilgewebe. Uber die Sta-dieneinteilung des Brustkrebses referiert Harmer, iiber Carcinoid-Tumoren Snow und iiber Sauerstoffbehandlung wahrend der Bestrahlung Churchill-Davidson, wah-rend Morrison iiber die Ergebnisse der Harnblasenbehandlung mit Supervolt-therapie berichtet. Auch die Chemotherapie kommt in 5 kurzen Aufsatzen zur Darstellung, und soziale Aspekte stehen im Vordergrund in den beiden Aufsatzen von Oettle und Lancaster. Das Buch ist vorziiglich ausgestattet und bildet eine groBe Hilfe fur den Krebstherapeuten, dem es nicht mehr moglich ist, alle Originalarbeiten zu studieren und dankbar ist fur gute kurze Zusammenfassungen.

H. R. Schinz, Zurich

Lenz, W.: Medizinische Genetik. Eine Einfiihrung in ilire Grundlagen und Probleme. Georg Thieme Verlag, Stuttgart 1961. VIII + 196 Seiten, 66 Abbildungen, 35 Tabellen. Kartoniert DM 23.-.

Die Humangenetik hat in den letzten Jahren groBe Fortschritte erzielt. Anstelle der oft vieldeutigen oder unzuverlassigen Stammbaumkasuistik sind biochemische und zytologische Analysen getreten. Ich erinnere an die Feststellung, daB der Mensch 46 und nicht 48 Chromosomen hat, ich erwahne die Entdeckung der Trisomie bei

326 Buchbesprechungen - Book Reviews - Livres Nouveaux 
mongoloider Idiotie, ferner die Aufklarung des Klinefelter-Syndroms als XXY-

Chromosomentypus - sterile Manner mit auBerem mannlichem Genitale. Gegen-stiick ist das Turner-UUrich-Syndrom - Individuen mit weiblichem auBerem Genitale, Gonadendysgenesie und verschiedenen MiBbildungen - mit der Chromosomen-formel XO. Zugrunde liegt diesen Storungen also eine falsche Chromosomenver-teilung in der Meiose. Sehr gut zusammengestellt sind die verschiedenen Hamo-globinopathien, die deshalb besonders bedeutungsvoll sind, weil sich einzelne For-men nur durch eine einzige Aminosaure unterscheiden - bei rund 300 Aminosauren des Globinanteils des Hamoglobins -, so daB man eine direkte Genwirkkette annehmen darf. Von den somatischen Mutationen wird auf Seite 120 gesprochen und betont, daB zwischen Mutationen und bosartigen Tumoren eine Reihe von Analogien bestehe. Ob der Mechanismus aber identisch ist mit einer Genmutation, bleibt frag-lich. Die «Mutation», die zur malignen Entartung der Zelle fiihrt, muB wohl eher den Vermehrungsmechanismus der Zelle selbst in Unordnung bringen und vielleicht erst sekundar das sich vermehrende genetische Material.

Das Buch ist sehr gescheit konzipiert und referiert, freilich in Auswahl, denneuesten Stand der Forschung. Von groBtem Nutzen ist es fiir den, der mit derklassischen Erblehre und der experimentellen Genetik gut vertraut ist. Die Aus-stattung ist eine vorziigliche. H. R. Schinz, Zurich

Medizinische Grundlagenforschung. Beirat: K. Bucher, Basel, W. Doerr, Kiel, A. Grumbach, Zurich, N. Henning, Erlangen, H. Holzer, Freiburg i. Br., A. Korn-mtiller, Gottingen, J. Kiihnau, Hamburg, H. Schaefer, Heidelberg, H. Schoen, Gottingen. Herausgegeben von Prof. Dr. K. Fr. Bauer, Erlangen. Band II. Bear-beitet von zahlreichen namhaften Fachgelehrten des In- und Auslandes. Georg Thieme Verlag, Stuttgart 1959. 827 Seiten, 187 Abbildungen, 63 Tabellen. DM 168.-.

Der zweite Band hat eine kleine Abanderung im Titel erfahren. Das Wort«Ergebnisse» ist gestrichen, die Beitrage sollen eine Mittelstellung einnehmen zwischen Ubersichtsreferat und Originalarbeit. Dies ist in den 17 Beitragen inhaltlichvollkommen gelungen. Auch auBerlich prasentiert sich das Buch wunderbar. DenKrebsforscher und Krebstherapeuten interessieren der Beitrag K. Fr. Bauer undE. Miiller: Die Zellenlehre; der Aufsatz V. Becker: Morphologisches Aquivalentbilddes auBeren und inneren Sauerstoffmangels; und vor allem die glanzende Darstellung der cancerogenen Stoffe durch Buu-Hoi (S. 465-550). Eine sehr erwiinschteErganzung zu diesem Abschnitt liefern Domagk und Mitarbeiter durch die Schilde-rung des heutigen Standes der Chemotherapie der Tumoren (S. 551-588). Ein gutesAutoren- und ein ausgiebiges Inhaltsverzeichnis bilden den SchluB dieses auBerstverdienstvollen Sammelwerkes.

H. R. Schinz, Zurich

Langreder, W.: Gynakologische Urologie. Mit einem Geleitwort von Prof. Dr. H. Schwalm. Georg Thieme Verlag, Stuttgart 1961. XII + 306 Seiten, 135 Abbildungen und 9 Tabellen. Ganzleinen DM 59.-.

Im Geleitwort von H. Schwalm, dem Direktor der Frauenklinik Wurzburg, wird betont, daB unbestritten manche Erkrankungen der Harnorgane bei der Frau von Gynakologen untersucht, behandelt und erforscht werden, hatte doch vor 23 Jahren Stoeckel schon eine gynakologische Urologie verfaBt. Das vorliegende Werk setzt diese Tradition fort, wobei der Gynakologe sich im Bereich der harnbildenden

Buchbesprechungen - Book Reviews - Livres Nouveaux 327

Organe kaum betatigt. Eine Ausnahme bilden nur die Schwangerschaftsnephro-pathien. Inhaltlich soil das vorliegende Werk, das 135 instruktive Skizzen enthalt und didaktisch sehr 
geschickt angeordnet ist, sowohl den urologischen Erforder-nissen einer modernen Frauenklinik als auch denen des Praktikers geniigen. Ent-scheidend fur die Stoffauslese war die Haufigkeit einer jeden Erkrankung und ihre prinzipielle Bedeutung. Das Kapitel X ist der Differentialdiagnose der urologischen Erkrankungen gewidmet, wahrend Kapitel XI stichwortartig die Symptomatologie behandelt. Den SchluB bildet eine Liste von Medikamenten, Kurorten und Heil-mittelgruppen. Gewidmet ist das Werk den Altmeistern der Gynakologie W. Stoeckel, H. Martins und R. Schroderf. Die Aufinachung ist vorziiglich. H. R. Schinz, Zurich Die Intersexualitat. Herausgegeben von Prof. Dr. C. Overzier. Georg Thieme Verlag, Stuttgart 1961. 560 Seiten, 193 Abbildungen, 38 Tabellen. Ganzleinen DM 119.-. Ein ausgezeichnetes Werk. Geschrieben wurde es von einem Kreis kundigster Fachleute, angefiihrt von C. Overzier, der sich nicht scheute, die Beitrage englisch schreibender Kollegen ins Deutsche zu iibertragen. Intersexualitat ist ein Problem, das bis vor kurzem zwar wohl fur einige Biologen und Mediziner von Interesse und sogar Lebensinhalt ihrer Forschung war, fur die meisten jedoch nur Kuriositatswert besaB. Dies ist schlagartig anders geworden, und mit der Erweiterung der Erkennt-nisse um das Wesen der Intersexualitat, mit der Moglichkeit, das genetische Ge-schlecht des Menschen festzustellen, Diskrepanzen zwischen Phanotyp und Genotyp aufzudecken und mit der Verbesserung klinischer Methoden hat sich die Inter-sexualitatsforschung zu einer hochaktuellen Arbeitsrichtung der Medizin entwickelt. Der heutige Stand des Wissens ist in dem vorliegenden Buch vortrefflich dargestellt, und in den verschiedenen Kapiteln sind nicht nur die umfangreiche Literatur ge-sichtet, sondern auchprazis und abgrenzend die wesentlichstenTatsachen zusammen-gefaBt.

liber die normale Entwicklungsgeschichte der Gonaden und der Geschlechts-gange berichtet M. Watzka. E. Witschi, bekannt durch seine Pionierstudien iiber die Geschlechtsbestimmung bei den Amphibien, stellt zusammen mit J. M. Opitz die Grundlagen der Intersexualitat dar, wahrend W. Koch Falle von Intersexualitat bei Saugetieren zusammenfaBt. Vom Entdecker des Geschlechtschromatins M. L. Barr selber stammt die ausgezeichnete Darstellung «Das Geschlechtschromatin», in welcher neueste Befunde verwendet sind, und ebenfalls von berufenster Seite, nam-lich von W. M. Davidson und D. R. Smith wird das Kerngeschlecht der Leukozyten in Wort und Bild demonstriert. Umfassend und von groBtem Interesse ist das Kapitel von C. E. Ford iiber die Zytogenese der Intersexualitat und Geschlechts-bestimmungen beim Menschen. In einem inhaltsreichen Beitrag, versehen mit wert-vollen Tabellen und Diagrammen, «Hormone und Intersexualitat» leiten J. Zander und H. D. Henning iiber zu den Darstellungen der klinischen Erscheinungsformen. Der Herausgeber C. Overzier bestreitet in meisterlicher Klarheit die Kapitel iiber «Hermaphroditismus verus», «Pseudohermaphroditismus», «Sogenanntes echtes Klinefelter-Syndrom», «Induzierter Pseudohermaphroditismus» und «Tumoren mit heterosexueller Aktivitat» (zusammen mit K. Hoffmann), wahrend G. A. Hauser zwei ausfuhrliche und wertvolle Beitrage iiber «Testikulare Feminisierung und Gonadendysgenesis» beisteuert, denen das Kapitel von J. R. Bierich iiber das «Adrenogenital Syndrom» nicht nachsteht. Die interessanten Beitrage von B. Lennox (Kerngeschlecht der Tumoren), von H. Gelbke (Plastische Operationen), 328 Buchbesprechungen - Book Reviews - Livres Nouveaux von R. Hasche-Kliinder (GenitalmiBbildungen ohne Intersexualitat) und vonJ. H. Schultz (Intersexualitat und Transvestitismus) runden das Bild vollends ab.Vorzuglich ist die Gesamtausstattung durch den Georg Thieme Verlag, ohne dienach Overzier «unser Vorhaben nie hatte verwirklicht werden konnen». Ein Vor-haben, das ausgezeichnet gegliickt ist und zu einem Werk gefiihrt hat, das warmstensempfohlen sei. Hedi Fritz-Niggli, Zurich 
Hess, W.: Erkrankungen der Gallenwege und des Pankreas. Diagnostik, Klinik und chirurgische Therapie. Mit Operationszeichnungen von Ingrid Schaumburg, Hamburg. Georg Thieme Verlag, Stuttgart 1961. XXIV + 672 Seiten, 267 Abbildungen in 560 Einzeldarstellungen. Ganzleinen DM 142.-.

Es ist auffallend, daB man iiber die Erkrankungen der Gallenwege und desPankreas ein 670 Seiten dickes Buch schreiben kann. So sehr hat sich die Wissen-schaft spezialisiert. Das Werk ist aber inhaltlich vorzuglich und ist hervorragendausgestattet. Wie in vielen neuen Biichern enthalt jede Seite 2 Spalten; dies giltaueh fur die Literaturverzeichnisse. Dies geschieht wohl, um Platz zu sparen. DerInhalt gliedert sich in folgende Hauptabschnitte: Anatomie und Physiologie, Patho-logie, Klinik der Gallenwege und des Pankreas, Diagnostik, interoperative Diagnostik, operative Technik, Operationswahl und Operationstaktik, Vorbereitung undAnasthesie bei Gallenwegsoperationen, postoperativer Verlauf und postoperativeKomplikationen, postoperative Beschwerden. Die Statistik umfafit 1654 eigeneFalle, darunter 1614 operative, von denen 1167 Nachsorgeberichte bis zu 12 Jahrenvorliegen. Das Buch ist Caroli, Paris, gewidmet, der seit 1942 schrittweise dieManometrie der Gallenwege vervollkommnet hat. Angaben iiber die Ergebnisse derFeinstrukturanalyse der verschiedenen Steinarten fehlen. In Abb. 180 wird gezeigt, was fur ein groBes Arbeitsteam die Durchfuhrung einer modernen Operation ver-langt. In Abb. 243 wird in perspektivischer Darstellung auf Grund einer Sammel-statistik die Absterbeordnung bei Pankreascarcinomen dargestellt. Nur 6\% diirftendie 5-Jahres-Grenze erreichen. Eigene Erfolgsstatistiken fehlen. Dies ist bedauerlich.Die Amerikaner sind uns Europaern heute in der exakten Berichterstattung derResultate weit voran. Den Leser interessiert nicht nur, was gearbeitet, sondern nochmehr, was geleistet worden ist. Die Abbildungen in dem Werke sind durchwegsprachtvoll. Fur Spezialisten ist das Werk unentbehrlich. H. R. Schinz, Zurich Janisch-Raskovic, W.: Das Carcinoma colli uteri, mit besonderer Beriicksichtigung der Strahlentherapie. Sammlung von Abhandlungen aus dem Gebiet der Frauen-heilkunde und Geburtshilfe. Neue Folge, Heft 20. Herausgeber: Prof. W. Stoeckel. VEB Georg Thieme, Leipzig 1960. 649 Seiten. DM 75.-.

Jede sorgfaltige und kritische Zusammenstellung der Erfolge und MiBerfolge am eigenen Krankengut und die Konfrontation mit den Erfahrungen Anderer ist wert-voll. Das trifft fur das vorliegende Buch, das dem Carcinoma colli uteri (C. c. u.) und seiner Prognose und Behandlung gewidmet ist, ganz besonders zu. In einem I. Teil werden die allgemeinen Richtlinien fur die Aufstellung einer Erfolgsstatistik besprochen, besonders eingehend die Gruppeneinteilung mit besonderer Beriicksichtigung der Gruppe 0 - die nach Auffassung des Referenten kein echtes Carcinom ist, denn die gefundenen epithelialen Veranderungen sind haufig reversibel; es handelt sich also - mit Ausnahme des echten invasiven Mikrocarcinoms - nieist um Pracancerosen. Teil II ist der Behandlung gewidmet, wie sie an der stadtischen Buchbesprechungen - Book Reviews - Livres Nouveaux 329 Frauenklinik in Chemnitz in den Jahren 1926-1948 durchgefiihrt wurde. Die Nach-kontrollen beziehen sich auf 2704 Falle. 1926-1930 war die Periode der vorwiegend operativen Verfahren, 1931-1935 die der elektiven Strahlentherapie und 1936-1948 die der ausschlieBlichen Strahlentherapie, abgesehen von wenigen Ausnahmen. Auf 2347 radiumbehandelte Collumkrebse betrug die durchschnittliche primare Strahlen-letalitat (nicht Mortalitat, wie irrtumlich angegeben) rund 1\%, stieg aber bei den fortgeschrittensten Fallen auf 2\%. Reversible Strahlenschadigungen wurden an der Blase an 5, im Rectum an 11 Fallen festgestellt. Auch 4 Rectumulcera heilten alle aus. In 13 Fallen stellten sich Genitalfisteln ein, von denen 8 geheilt wurden. Die iibrigen verliefen todlich. Andere Komplikationen sind selten. 
Die absolute Leistung (5-Jahres-Heihmgen) bei den Nur-Bestrahlten fiir alle Gruppen zusammen fiir die Periode 1945-1948 betrug 35\% (173:502), die relative Leistung in Gruppe I 63\% (33:52), in Gruppe II 44\% (79:180), in Gruppe III 26\% (61:238) und in Gruppe IV 0\% (0:32).

Der III. Teil ist der Erorterung des C. c. u. bei besonderen physiologischen und pathologischen Zustanden der Genitalien und des Gesamtorganismus gewidmet. In Stichworten sei der Inhalt angedeutet: C. c. u. bei Schwangeren, bei weiteren Ge-schwiilsten am Uterus and an anderen Uterusabschnitten, bei UterusmiBbildungen, bei Syphilitikerinnen, bei Diabetes usw. Diese Ausfuhrungen sind auBerordentlich wertvoll fiir den Krebstherapeuten und dienen gegebenenfalls zum Nachschlagen und Nachlesen. Die Ausstattung des Buches laBt zum Teil etwas zu wiinschen iibrig.

H. R. Schinz, Zurich Antibiotica et Chemotherapia. Fortschritte - Advances - Progres. Vol. 9. Hgb. von 0. Gsell und R. L. Mayer. Verlag S. Karger, Basel/New York 1961. IV + 192 S., 32 Abb. sFr. 40.-. Die bis jetzt erschienenen 9 Bande sind fiir den Radiotherapeuten unentbehrlich, geben sie ihm doch Einblick in die Fortschritte der Chemotherapie mit Antibiotikaund Sulfonamiden. Fast jeder Krebsfall leidet auch an Sekundarinfektionen, die eszu bekampfen gilt. Daruber hinaus erfahrt man Wichtiges iiber die Versuche, malig-ne Erkrankungen durch Chemotherapeutika zu heilen oder doch deren Wachstumund deren Ausbreitung im Korper zu stoppen. Auch iiber Knochenmarktransplan-tationen und iiber interne Radioisotopentherapie wurde berichtet. Im vorliegenden9. Volumen bearbeiten unter dem Redaktionskomitee von 0. Gsell, Basel undR. L. Mayer, Washington D.C. 6 Autoren einschlagige Themata, 2 Aufsatze sinddeutsch, 2 englisch geschrieben. Frisk, Stockholm, gibt einen Riick- und Ausblickiiber 25 Jahre Chemotherapie mit Antibiotici und Sulfonamiden. Neipp und Mit-arbeiter berichten iiber experimentelle Ergebnisse mit Sulfonamiden, wahrendMiickter die Pharmakologie der basischen Streptomyces-Antibiotica behandelt.Schnitzer schildert den heutigen Stand der Chemotherapie der Lepra und derTuberkulose im Experiment. Wir empfehlen die Anschaffung der ganzen Serie angelegentlich. H. R. Schinz, Zurich

Muntean, E.: Die preoperative Rontgenbestrahlung des Mammakarzinoms. Georg Thieme Verlag, Stuttgart 1961. IV + 108 Seiten, 9 Abbildungen, 26 Tabellen. Kartoniert DM 17.50. Wenn wir Krebstherapeuten fragen, was das beste Verfahren sei bei Behandlung des Brustkrebses, so erhalten wir sehr verschiedene Antworten. Es gibt Chirurgen, 330 Buchbesprechungen - Book Reviews - Livres Nouveaux welche nur operieren und die Nichtoperablen der Bestrahlung iiberweisen. Es gibtandere, welche operieren und nachbestrahlen lassen, und es gibt Radiotherapeuten, welche in alien Stadien nur bestrahlen. Neuerdings ist auch die Rontgenvorbe-strahlung in den Vordergrund getreten. Muntean hat das Verdienst, die Erfahrungenbei dieser Methode zusammengestellt zu haben, und er stiitzt sich dabei auch aufeigene Ergebnisse. Fur preoperative Bestrahlung eignen sich Grenzfalle und primarinoperable Tumoren, die operabel werden. Bei operablen Fallen wird ein Teil derCarcinomzellen vernichtet. Vor sehr hohen Strahlendosen ist aber zu warnen. Eskommt nicht nur auf die Reaktion des Tumorgewebes an, sondern auch auf die Be-einfiussung des Tumorbettes. Die Zahl der Fruhrezidive ist nach dem Autor geringer.Die Uberlebensrate nach 5 Jahren betragt bei der praoperativen Bestrahlung40-60\%. Es handelt sich dabei lediglich um die Prozentzahlen fur das gesamteMaterial. Nur sehr wenige Autoren geben Erfolgsstatistiken fur die einzelnenStadien. Sicher ist durch die preoperative Bestrahlung eine eindeutige Besserungder 5Jahres-Ergebnisse bei den fortgeschrittenen Fallen des Stadiums II erreichtworden. Die 
preoperative Bestrahlung ist nach dem Autor die Methode der Wahlbei den fortgeschrittenen Stadien. Das sorgfaltige Literaturverzeichnis umfaBt880 Nummern. H. R. Schinz, Zurich Memoirs of the Society for Endocrinology, No. 10. Progress in Endocrinology, Part II. Biochemistry and Biological Actions of Steroids and other Hormones. Edited by K. Fotherby, J. A. Loraine, J. A. Strong and P. Eckstein. Cambridge University Press 1961. XII + 127 p., ill. Price 45 s net.

Die Krebstherapie ist insofern in Bewegung, als neben die Chirurgie und dieStrahlenbehandlung zusatzliche Hormonzufuhren oder Hormonausschaltungen an-gewendet werden. Die klinischen Untersuchungen bediirfen einer Erganzung durchdie experimentelle Forschung, welche den HormoneinfiuB auf Experimental-tumoren, auf Heterotransplantate usw. analysiert. Auch die Hormonausscheidungdurch den Urin bei Krebskranken nach Adrenalektomie oder Oophorektomie usw.muB gepriift werden. Solchen Fragen sind die 14 Aufsatze gewidmet unter demStichwort: «Are the endocrine glands directly related to Cancer ?». Die vorliegendeZusammenstellung ist auBerst wertvoll und wird hoffentlich bald eine weitere Fortsetzung finden. H. R. Schinz, Zurich Cell Physiology of Neoplasia. A collection of Papers presented at the Fourteenth Annual Symposium on fundamental Cancer Research, 1960. Published for the University of Texas M.D. Anderson Hospital and Tumor Institute. University of Texas Press, Austin 1960. 653 Seiten. Man riickt heute den Lebensaufierungen von Krebszellen mit alien modernen Methoden der Physik, der Chemie und der Biologie auf den Leib. Das Einzelwissen um diese Vorgange hat auBerordentlich zugenommen, und wir Kliniker sind den Gelehrten, welche auf dem Gebiete der Grundlagenforschung des Krebses tatig sind, fur die Ubermittlung ihrer neuesten Resultate zu groBem Dank verpflichtet. Dies gilt besonders fur das umfangreiche Werk von 650 Seiten, das unser gegenwartiges Wissen iiber die Zellphysiologie neoplastischer Zellen zusammenfaBt. Es handelt sich dabei um das 14. jahrliche Symposium iiber Krebsforschung 1960 in den USA. Die Zusammenstellung, an der hervorragende Gelehrte beteiligt sind, verdanken wir dem Staff of the University of Texas, M.D. Anderson Hospital and Tumor

Buchbesprechungen - Book Reviews - Livres Nouveaux 331

Institute. Das erste Symposium wurde 1946 abgehalten, und seit 1952 wird jeweilsein zentrales Thema gewahlt. So z. B. die Gewebekultur, Krebs und Vererbung usw.Fiir 1960 lautete das zentrale Problem die Zellphysiologie der Neoplasien. UberChromosomenbefunde in Tumorgewebe berichtet P. C. Roller, iiber Geschlechts-chromosomen eine ganze Arbeitsgruppe von der Harvard Medical School. Einweiterer Aufsatz ist den Nukleolen der Malignomzelle gewidmet (M. J. Kopac), und M. L. Watson stellt die Ergebnisse der Elektronenmikroskopie der Kerne zu-sammen. Dann folgen Aufsatze iiber das Cytoplasma, darunter einer iiber denGolgiKomplex von A. T. Dalton vom National Cancer Institute in Bethesda.Elektronenmikroskopische Untersuchungen von Virustumoren stammen von einerArbeitsgruppe aus Texas. Der Bericht von Novikoff iiber Enzymlokalisation inTumorzellen ist das Resultat des Departement of Pathology am Albert EinsteinCollege in New York. Nach diesen mehr morphologisch orientierten Arbeiten iiberKern und Plasma folgen Mitteilungen iiber den Stoffwechsel von D.N.A. undR.N.A., die im Vordergrund der Forschung stehen, seitdem im Oktober 1959 Ochoaund Kornberg fiir deren Synthese den Nobelpreis erhalten haben. Es ist nicht mog-lich, naher auf alle diese wichtigen Publikationen einzugehen, und ich beschrankemich darauf, daG 6 weitere Arbeiten dem Zellwachstum und der Zelldifferenzierungvon Krebszellen gewidmet sind, wobei entwicklungsphysiologische Probleme imVordergrund stehen. Jeder Abschnitt enthalt ein Verzeichnis der 
modernstenangelsachsischen Literatur, und den SchluB macht ein guter Index. Solche zusammenfassende Berichte unter einem Zentralproblem sind in Europa besonderserwiinscht, wenn wir auch mit einer gewissen Betriibnis feststellen miissen, daG dieeuropaische einschlagige Literatur fast vollstandig vernachlaBigt wird, falls dieOriginalarbeiten nicht englisch abgefaBt sind. $\quad$ H. R. Schinz, Zurich Spezielle Pathologic fiir die klinische und pathologische Praxis (A Text on Systemic Pathology). Von 0. Saphir, Chicago. Deutsche Ubersetzung von F. Kessler, Miinchen und K. F. Wellmann, Ottawa/Canada. Georg Thieme Verlag, Stuttgart 1961. 335 S., 330 Abb. Preis Ganzleinen DM 164.--

Es liegt ein auBerordentlich nutzliches Buch vor, und wir sind den Ubersetzern und dem deutschen Verleger zu groBem Dank verpflichtet. Der Student wird das Werk eingehend und mit Erfolg studieren, wenn er an der speziellen Pathologie Freude hat und sich griindlich informieren will. Dem Krankenhaus-Pathologen, dem Kliniker und dem Krebsspezialisten ist es dienlich, auch er kann sich rasch und zuverlassig iiber ein bestimmtes Gebiet orientieren. Einseitigkeit ist vermieden. Der vorliegende erste Band umfafit 10 Kapitel. Bei der Erorterung des Herzens werden auch die Geschwiilste angefiihrt und mit ausgezeichneten Abbildungen belegt. Es folgt hier wie in alien anderen Kapiteln ein zuverlassiges Literaturverzeichnis, das z. B. beim Herzen 364 Nummern umfafit. Es werden aber im allgemeinen nur angelsachsische Arbeiten angefiihrt. Die angeborenen Herzleiden sind griindlich behandelt, sind sie doch in den letzten Jahren mit Erfolg operativ angegangen worden. Auch hier findet sich ein Literaturverzeichnis mit 373 Nummern. Die Lungengeschwiilste finden sich auf Seite 315-336. Erfreulich ist, daB nicht nur rein histologische und makroskopische Schilderungen zu finden sind, sondern auch An-gaben iiber die klinische Bedeutung, iiber Fruhsymptome (Bronchialadenome, Zylindrome), ferner Mitteilungen iiber die Zunahme der Lungenkrebse und die all-ffillige GefShrdung durch Inhalieren von Zigarettenrauch und durch andere Luft-

332 Buchbesprechungen - Book Beviews - Livres Nouveaux

verschmutzung. Uberall findet sich eine kritische und vorsichtige Stellungnahme.Im Kapitel «Harnsystem» werden zuerst die gutartigen epithelialen Geschwiilsteund Nebennierenreste besprochen. Das Hypernephrom, d. h. eine Nebennieren-geschwulst, die in der Niere entsteht, wird Nierencarcinom oder Nephrom genanntund ausgefuhrt, daB iiber die renale Abkunft heute keine Zweifel mehr bestehen, sei es, daB diese Nierencarcinome von den Tubuli, sei es, daB sie von Adenomen aus-gehen. Auch hier finden sich interessante Erorterungen fiber die klinischen Befunde, die Metastasierung, die cytologische Diagnose usw. Es folgt die Schilderung desWilms-Tumors (Adenosarkom der Niere, Nierenmischtumor, embryonales Nephromund Nephroblastom). Wahrscheinlich gebe es auch echte Teratome. Gutartigerenale Bindegewebsgeschwfilste werden dargestellt, sie sind haufig, bosartigekommen selten vor. Als weiteres Beispiel zitiere ich die Einteilung der Ovarial-tumoren. Sie ist kurz und didaktisch geschickt. Es werden unterschieden: 1. cysti-sche gutartige und bosartige Geschwiilste, einschlieBlich Endometriome und Teratome, 2. solide gutartige und bosartige Geschwiilste bindegewebiger und epithelialerNatur mit Ausnahme der besonderen Tumorformen, 3. besondere Geschwiilste, wahrscheinlich dysontogenetischen Ursprungs mit embryonalen Resten und 4.metastatische bosartige Geschwiilste. Unter den besonderen Geschwulsten werdenaufgezahlt Dysgerminome, die immer maligne sind, Granulosazelltumoren undThekazelltumoren, die beide oft als feminisierende Mesenchymome bezeichnetwerden, die Androblastome, die aus der testikularen Anlage des Ovars entstehensollen, wobei manchmal jeder endokrine Effekt fehlt, manchmal wirken sie ver-mannlichend, selten verweiblichend. 
Dazu gehoren auch die sogenannten Arrheno-blastome, auch hier finden sich viele klinische Angaben. Einzelheiten miissen demvorziiglich ausgestatteten Werk entnommen werden. Es sei warmstens zur An-schaffung empfohlen. H. R. Schinz, Zurich

Varia

Die Bibliothek des Forschungs-Institutes fur experimentelle Medizin und Chirur-gie der Universitat Montreal ist durch Feuer groBtenteils zerstort worden. Dm sie wieder aufzubauen, mochten wir an die Leser der

Zeitschrift Oncologia

die Bitte richten, uns von alien ihren Veroffentlichungen 1-2 Sonderabdrucke zu schicken, soweit sie noch erhaltlich sind, besonders solche aus den Gebieten der Endokrinologie und der Stress-Forschung.

S. Sonderabdrucke unserer Veroffentlichungen konnen wir von nun an nur noch auf Anforderung verschicken da unsere umfangreiche Adessensammlung ebenfalls vernichtet wurde an Hand der wir bisher regelmaBig Sonderabdrucke der neuesten Veroffentlichungen verschickten.

Hans Selye

Professor und Direktor

Institut de Medecine et de Chirurgie experimentales Universite de Montreal Case postale 6128 Montreal

Canada. 